\title{
Green's Functions in Lossy Layered Media: Integration Along the Imaginary Axis and Asymptotic Behavior
}

\author{
Juan R. Mosig, Fellow, IEEE, and Alejandro Alvarez Melcón, Member, IEEE
}

\begin{abstract}
This paper presents an efficient technique for evaluating Green's functions associated to layered media, when formulated as Sommerfeld integrals in the space domain. The key step in the formulation is that Sommerfeld integrals are computed choosing a suitable integration path which is closed through the imaginary axis of the complex spectral plane. It is shown that with this original choice of the integration contour, the numerical effort usually involved in the evaluation of Sommerfeld integrals can be greatly reduced, specially when large source-observer distances are involved. One asset of this technique is that it can be easily incorporated into integral equation based $C A D$ packages for the efficient analysis of complex printed microwave circuit and antennas. In addition, the theoretical developments needed to set up the numerical algorithm throw a new light on the asymptotic behavior of the layered media Green's functions for large source-observer distances.
\end{abstract}

Index Terms-Asymptotic behavior, integral equation, multilayered Green's functions, printed antennas, printed circuits.

\section{INTRODUCTION}

$\mathbf{P}$ RINTED antennas and associated circuits have been extensively investigated in the last decades. Among the currently used models, methods based on integral equation techniques, formulated both in the spectral and in the space domain, are specially attractive, since they provide excellent accuracy and good computational speed. Details of space domain integral equations techniques applied to the analysis of infinite multilayered printed structures can be found for instance in [1]. Of paramount relevance in this formulation is the concept of Green's function, defined as the fields or potentials created by a point unit source embedded in a layered medium (Fig. 1).

The developments in this paper are based on previous works by one of the authors [2]-[4]. In those works, the relevant spatial domain Green's functions were formulated as Sommerfeld integrals of the corresponding spectral domain counterparts, and the accurate numerical evaluation of these Sommerfeld integrals was shown to be a critical point.

For the numerical evaluation of Sommerfeld integrals several approaches have been developed in the past. Traditionally, integration through the real axis combined with pole extraction techniques and averaging methods has been employed, leading to very efficient algorithms [3]. The original Sommerfeld integration path along the real axis has also been successfully closed

Manuscript received September 3, 1997; revised January 29, 2003.

The authors are with the Laboratoire d'Electromagnetisme et d'Acoustique, Ecole Polytechnique Fédérale de Lausanne, Lausanne CH-1015, Switzerland (e-mail: alejandro.alvarez@upct.es).

Digital Object Identifier 10.1109/TAP.2003.820946

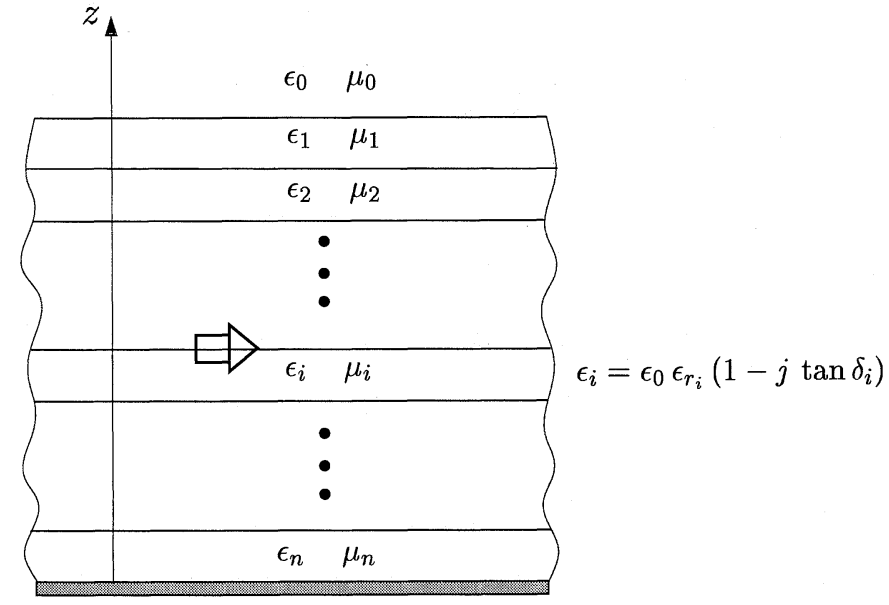

Fig. 1. General multilayered structure analyzed in this paper.

at infinity, thus reducing the whole integration path to the contributions of the poles and branch cuts of the spectral domain Green's functions [5]-[7]. In addition, other authors have deformed the real axis into a path going through the upper half complex plane to avoid the poles of the spectral domain Green's functions, using rectangular contours [8], or alternatively elliptic integration paths [9].

While all these techniques are indeed efficient for relatively small source-observer distances, they usually require the integration of functions exhibiting abrupt variations and fast oscillating behaviors when large source-observer distances are involved [2], [10]. However, many current practical problems involve distances of several tens or even hundreds of wavelengths. This is the case when computing mutual coupling inside large arrays [11], [12], or when modeling cavity backed antennas by using space images respect to the cavity's lateral walls [13].

To solve this problem, analytical techniques have been developed in the past to express the Green's functions in closed form expressions which are valid for large source-observer distances [14], [15]. More recently, these results have been used in the study of the coupling between two dipole antennas in [12]. In that work the analytical formulation of the Green's functions in surface and space waves allowed to get insight into the coupling mechanisms occurring between two printed dipoles, when the basic substrate parameters (thickness and dielectric constant) are varied. While these techniques are indeed accurate and efficient, however, they are only valid for particular 
layered structures, and new expressions must be developed for new layered configurations, hence with loss of generality. An alternative and very popular technique to obtain the spatial domain Green's functions in closed form is the so called complex image technique first developed in [16]. The approach is based on representing the spatial domain Green's functions as the sum of three contributions, namely, a quasistatic images term dominating in the near field region, the surface waves contribution, and a contribution from the so called complex images which is related to leaky waves. Due to the exponentially decaying part of the complex image series, the convergence behavior of the technique is excellent [17], [18].

Another interesting technique for the numerical evaluation of the Sommerfeld integrals was derived in [2], and it consisted on a new choice of the integration contour which was closed through the imaginary axis of the spectral plane. However, the imaginary axis algorithm developed in [2] is only valid for lossless layers. Related to this technique, it is interesting to mention the work derived in [10], which utilized the imaginary axis algorithm to develop a series representation of the Green's functions, by using the Bessel function argument-multiplication theorem. An additional advantage of the technique in [10] is that the dependence with the source-observer distance $(\rho)$ is extracted from the basic Sommerfeld integrals, so that numerical integration need not to be repeated for all $\rho$ 's. The technique derived in [10], although efficient, remains valid only for lossless layers, since it is based on the original technique described in [2].

In this paper the basic technique described in [2] is extended and generalized to the case of lossy dielectrics. Once the formulation is derived, all the advantages of the imaginary axis integration method can be effectively set up to work for the analysis of circuits and antennas printed on complex stratification configurations, containing an arbitrary number of lossy dielectrics. The theoretical developments needed to set up the numerical algorithm throw a new light on the asymptotic behavior of the multilayered media spatial Green's functions. In this paper, a thorough discussion on this asymptotic behavior is included together with the numerical results obtained for the Green's functions using the developed approach. A very interesting and somewhat complementary discussion on the asymptotic behavior of Green's functions can be found in [12], but derived in the specific context of the mutual coupling between two printed dipole antennas.

\section{BASIC THEORY}

A general form for a Green's function (Sommerfeld integral) associated to the problem of Fig. 1 is [3]

$$
G(\rho)=S_{n}[\tilde{G}]=\int_{0}^{\infty} J_{n}\left(k_{\rho} \rho\right) k_{\rho}^{n+1} \tilde{G}\left(k_{\rho}\right) d k_{\rho}
$$

where $J_{n}$ is the Bessel function of order $n$, and $\tilde{G}\left(k_{\rho}\right)$ a generic spectral Green's function which can be obtained analytically for a layered medium [3].

To solve (1), traditional integration techniques along the real axis define the branch cut of $\tilde{G}\left(k_{\rho}\right)$ with the typical hyperbolic cuts described in [2]. If the selected integration path is a pure real axis contour, then the residue theorem leads to the following integral decomposition for (1) [2]

$$
\begin{aligned}
S_{n}[\tilde{G}] & =\int_{0}^{\infty} J_{n}(\xi \rho) \tilde{G}(\xi) \xi^{n+1} d \xi \\
& -\pi j \sum_{i} J_{n}\left(k_{\rho_{i}} \rho\right) R_{i} k_{\rho_{i}}^{n+1}=T_{1}+T_{2}
\end{aligned}
$$

with $k_{\rho}=\xi+j \eta$. In above expression $k_{\rho_{i}}$ is the $i$ th pole of the spectral domain function, $R_{i}$ its residue, and the bar in the integration symbol indicates that the integral is to be considered as a Cauchy principal value. Moreover, the first term $T_{1}$ is an improper integral, extended to the unbounded interval $[0, \infty]$, of a function involving the highly oscillatory Bessel function $J_{n}$. It is clear that the oscillating behavior makes quite difficult the numerical evaluation of this integral, and this situation worsens when the transverse distance increases as recognized in [2], [10].

The principal value in this $T_{1}$ term can be numerically handled with techniques such as the folding around the pole method [2]. Alternatively, the integration path can be brought out of the real axis by contour deformation, using for instance an elliptic path [9]. This strategy avoids the poles [term $T_{2}$ in (2)] which then do not need to be computed.

An alternative way of computing the Sommerfeld integral was derived in [2], and it consisted on closing the integration path through the imaginary axis. The formulation presented in [2], however, is only valid for lossless dielectric layers, and expresses the Sommerfeld integral in the following three terms:

$$
\begin{aligned}
S_{n}[\tilde{G}]= & j \int_{0}^{k_{0}} H_{n}^{(2)}(\xi \rho) \operatorname{Im}[\tilde{G}(\xi)] \xi^{n+1} d \xi \\
& -\frac{2}{\pi} \int_{0}^{\infty} K_{n}(\eta \rho) \operatorname{Im}[\tilde{G}(j \eta)] \eta^{n+1} d \eta \\
& -\pi j \sum_{i} H_{n}^{(2)}\left(k_{\rho_{i}} \rho\right) k_{\rho_{i}}{ }^{n+1} R_{i}=T_{1}+T_{2}+T_{3} .
\end{aligned}
$$

This expression used explicitly the fact that for lossless media the spectral Green's function $\tilde{G}(\xi)$ is purely real in the real axis for $\xi>k_{0}$. However, in a lossy case $\tilde{G}(\xi)$ is complex everywhere.

In the present work we have extended the work derived in [2] so that lossy layers can be accounted for in the formulation. To do so, the integration contour has been modified as shown in Fig. 2, so that now the path is closed through both the positive and negative imaginary axis. Using the new integration contour, Sommerfeld integral can be written as follows:

$$
\begin{aligned}
S_{n}[\tilde{G}]= & \frac{1}{2} \int_{0}^{k_{0}} H_{n}^{(2)}(\xi \rho)\left[\tilde{G}_{1}(\xi)-\tilde{G}_{2}(\xi)\right] \xi^{n+1} d \xi \\
& +\frac{j}{\pi} \int_{0}^{\infty} K_{n}(\eta \rho)[\tilde{G}(+j \eta)-\tilde{G}(-j \eta)] \eta^{n+1} d \eta \\
& -\pi j \sum_{i} H_{n}^{(2)}\left(k_{\rho_{i}} \rho\right) k_{\rho_{i}}{ }^{n+1} R_{i}=T_{1}+T_{2}+T_{3} .
\end{aligned}
$$




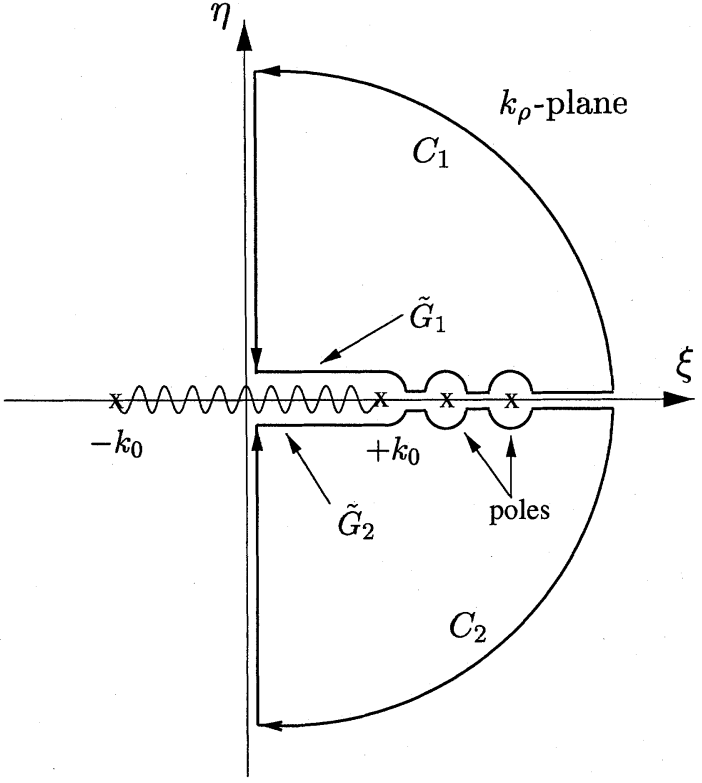

Lossless Situation.

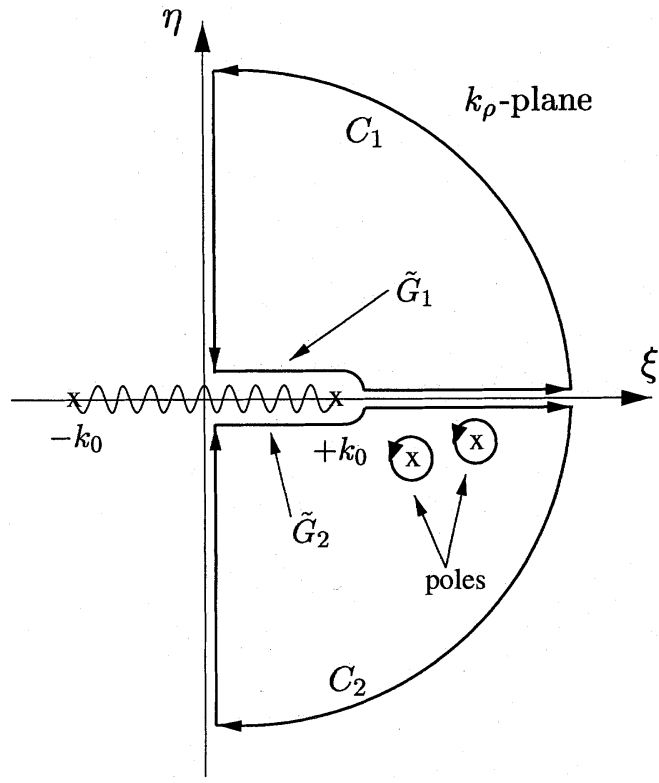

Lossy Situation.

Fig. 2. Complex spectral plane showing the alternative integration contours through the imaginary axis for the evaluation of Sommerfeld integrals.

where $\tilde{G}_{1}(\xi)$ is the spectral domain Green's function in the real axis above the branch cut, and $\tilde{G}_{2}(\xi)$ is the spectral domain Green's function in the real axis below the branch cut (see Fig. 2).

In a lossless situation, the spectral domain Green's function $\tilde{G}\left(k_{\rho}\right)$ satisfies the following two relations:

$$
\tilde{G}_{1}(\xi)=\tilde{G}_{2}^{*}(\xi), \quad \tilde{G}(+j \eta)=\tilde{G}^{*}(-j \eta)
$$

and the star symbol is used to denote complex conjugate. If above relations are introduced in (4), then the original formula (3) for lossless layers is obtained.

\section{NUMERICAL DETAILS}

Based on the formulation of (4), efficient computer codes can be built for the evaluation of the Sommerfeld integral for large source-observer distances. To demonstrate this fact, we will now explore some numerical techniques which have been applied to the evaluation of all three terms shown in (4).

The first term $T_{1}$ is an integral over the bounded interval [0, $k_{0}$ ] of a function without singularities in the integration interval. Since the integration interval is bounded, the evaluation of this $T_{1}$ term presents, in principle, no difficulties from the numerical point of view. The only minor problem is due to a removable singularity in $k_{\rho}=k_{0}$, originated by the presence of the branch point at $k_{0}$. In Fig. 3(a) we present a typical function to be integrated showing smooth behavior, but which exhibit a singularity in the derivative near $k_{\rho}=k_{0}$ due to the branch point. Knowing that the branch point is due to the term $\left(1 / \sqrt{k_{0}^{2}-k_{\rho}^{2}}\right)$, the weak singularity introduced can be easily removed by making a well known change of variables involving the sine function [19], thus obtaining the following alternate expression:

$$
\begin{aligned}
T_{1}=\frac{1}{2} \int_{0}^{\pi / 2} H_{n}^{(2)}(\theta \rho)\left[\tilde{G}_{1}(\theta)-\tilde{G}_{2}(\theta)\right] k_{0}^{(n+2)} \\
(\sin \theta)^{n+1} \cos \theta d \theta
\end{aligned}
$$

and now the function to be integrated is shown in Fig. 3(b). It is clear that the singularity has been effectively extracted, and that the behavior of the function to be integrated is smoother around the branch point $k_{0}$.

Finally, the last numerical difficulty concerning this $T_{1}$ term is due to the oscillating nature of the Hankel function, and to the fact that the integrand oscillates faster for larger sourceobserver distances. However, since the integration interval is finite, an efficient numerical algorithm can be constructed based on the quasiperiodicity of the Hankel function. In this study we have observed that good numerical precision is achieved taking seven points per "period" of the Hankel function. Following this rule, the final number of integration points $N_{p}$ for a given source-observer distance $\rho$ is computed with the following direct calculation:

$$
N_{p}=\frac{7 k_{0} \rho}{2 \pi} .
$$

Next, the term $T_{2}$ is an improper integral extended to the unbounded interval $[0, \infty]$ along the imaginary axis of the complex $k_{\rho}$ plane. It is important to note that the function to be integrated does not exhibit an oscillatory behavior. In consequence, standard numerical techniques based on oscillating functions such as the weighted average method [2], [3] will not work for this integral. However, thanks to the fast decaying behavior of the modified Bessel function it is expected that simple numerical 


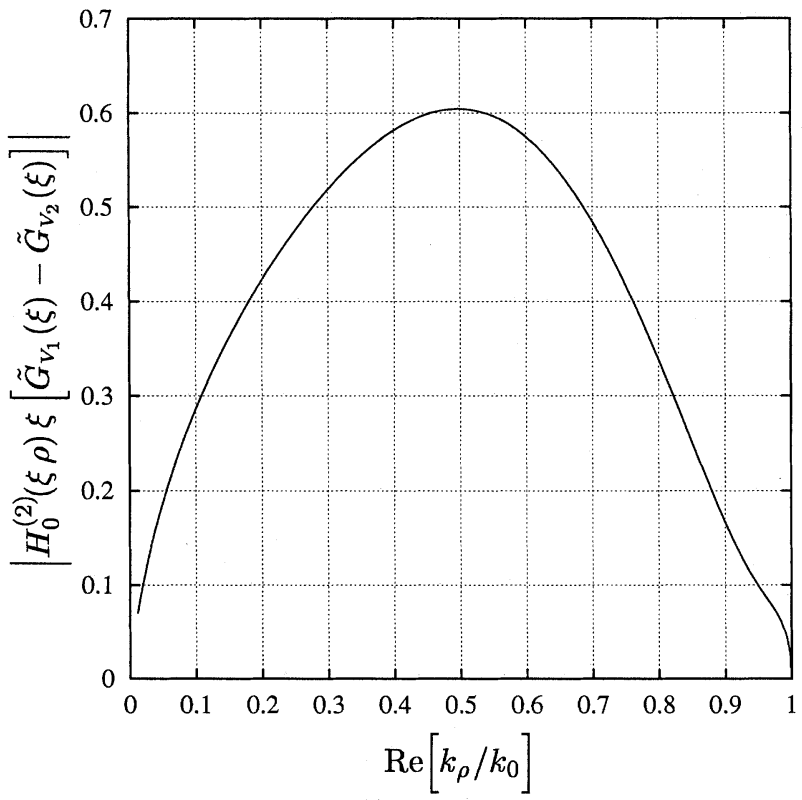

(a)

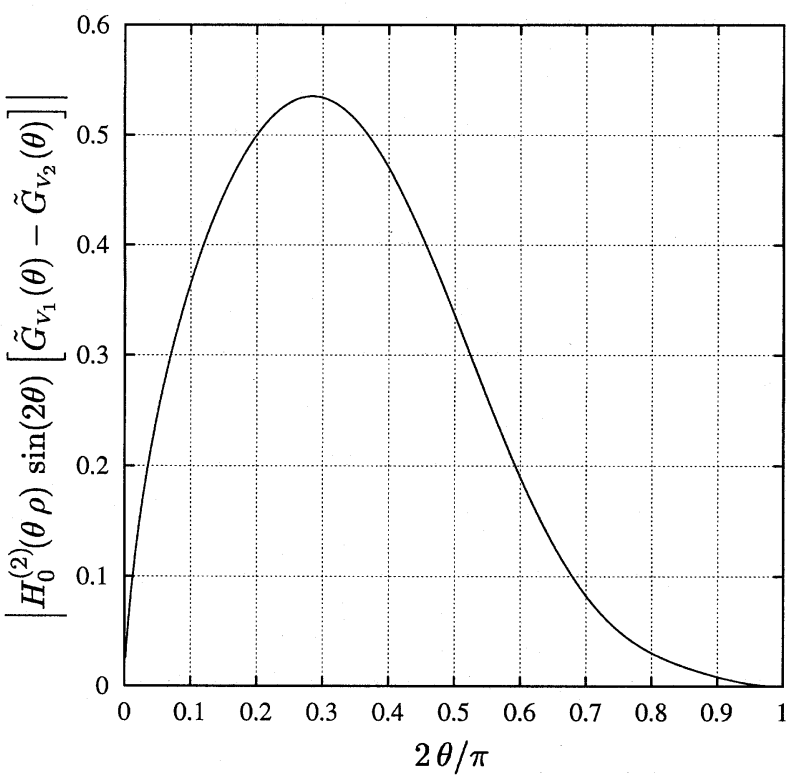

(b)

Fig. 3. Function to be integrated for the evaluation of $T_{1}$. The structure contains three layers: $\epsilon_{r_{1}}=9.0, h_{1}=4.0 \mathrm{~mm}, \epsilon_{r_{2}}=7.0, h_{2}=3.0 \mathrm{~mm}$ $\epsilon_{r_{3}}=5.0, h_{3}=1.0 \mathrm{~mm}$. Losses are: $\tan \delta=0.02$ for all layers and frequency is $10 \mathrm{GHz}$. (a) No singularity extraction and (b) with singularity extraction.

techniques converge very fast. To evaluate this integral a technique based on the reduction of the unbounded interval $[0, \infty]$ to the unitary interval $[0,1]$ is employed. The main advantage of this method is that the resulting integral is extended over a finite interval, and thus, it can be computed using standard numerical techniques. The method starts by splitting the whole unbounded interval into two subintervals: $[0, \infty]=[0, b]+[b, \infty]$, so that the integral $T_{2}$ is expressed as

$$
\begin{aligned}
T_{2}= & \frac{j}{\pi}\left\{\int_{0}^{b} K_{n}(\eta \rho)[\tilde{G}(+j \eta)-\tilde{G}(-j \eta)] \eta^{n+1} d y\right. \\
& \left.+\int_{b}^{\infty} K_{n}(\eta \rho)[\tilde{G}(+j \eta)-\tilde{G}(-j \eta)] \eta^{n+1} d y\right\}
\end{aligned}
$$

where $b$ is an arbitrary value. The following two changes of variables are then applied, respectively, to the first and second integrals in (8)

$$
\begin{array}{ll}
\eta=b t, & d \eta=+b d t \\
\eta=\frac{b}{t}, & d \eta=-\frac{b d t}{t^{2}}
\end{array}
$$

so that (8) transforms into (10), shown at the bottom of the page. Equation (10) can now be solved using standard GaussLegendre quadrature formulas in the interval $[0,1]$. It is also interesting to note that the technique will be efficient only if $K_{n}$ tends to zero faster that the rest of the function tends to infinity when $t \rightarrow 0$. This will be certainly the case since the asymptotic expression of the modified Bessel function $K_{n}$ for large arguments contains a decaying exponential of the type $[\exp (-n / t)]$, which largely compensate for the singularity of the term $1 /\left(t^{n+3}\right)$ appearing in (10).

Another interesting aspect of this method is that the term $(b \rho)$ is part of the argument to the modified Bessel function $K_{n}$, and it is convenient to take a constant value for all possible spatial distance values. In consequence an auxiliary parameter $\alpha$ can be defined, so that in each case the suitable value of the interval parameter $b$ is computed with the following direct calculation:

$$
b=\frac{\alpha}{\rho} .
$$

For the practical implementation of this technique several values of the auxiliary $\alpha$ parameter have been tried, observing good numerical convergence of the algorithm for an optimum value of $\alpha$ in the range: $1 \leq \alpha \leq 5$. With all these cautions, the numerical accuracy obtained with this technique is very satisfactory.

Finally, the term $T_{3}$ is expressed as a sum of surface wave contributions directly linked with the poles of the spectral domain Green's functions. The computation of poles in a general lossy multilayered structure is a delicate task, but can be accomplished accurately by using the analytical properties of the spectral Green's functions [20]. In this work an efficient algorithm has been developed. First the poles are located on the real axis for the lossless case. Then they are tracked as they penetrate into the lower half complex plane (Fig. 2) reacting to the introduction of losses. The whole procedure is a straightforward generalization of the technique described in [2].

$$
T_{2}=\frac{b j}{\pi} \int_{0}^{1}\left\{K_{n}(b t \rho)[\tilde{G}(+j b t)-\tilde{G}(-j b t)](b t)^{n+1}+K_{n}\left(\frac{b}{t} \rho\right)\left[\tilde{G}\left(+j \frac{b}{t}\right)-\tilde{G}\left(-j \frac{b}{t}\right)\right] \frac{\left(\frac{b}{t}\right)^{n+1}}{t^{2}}\right\} d t .
$$




\section{ASYMPTOTIC BEHAVIOR}

In addition to provide an interesting way for numerically computing the Green's functions, the decomposition of any Sommerfeld integral into the three terms of (4) gives us some useful theoretical insights concerning the behavior of Green's functions in the near and far field regions.

Although we have found convenient for numerical purposes to make a distinction between terms $T_{1}$ and $T_{2}$, it is evident that the combination $T_{1}+T_{2}$ represents the integration along the traditional hyperbolic cuts, as can be easily demonstrated by contour deformation [7]. The relevant fact is that $T_{1}$ (Hankel integral) contains the branch point contribution, and therefore, corresponds to the space wave, while $T_{2}$ (Modified Bessel integral) including the point at infinity is associated with a quasistatic behavior [2]. Traditionally, the combination of $T_{1}$ and $T_{2}$ has been known as the continuous-spectrum field. More precisely, $T_{1}$ alone (lossless case) or combined with $T_{2}$ (lossy case) shows the typical asymptotic behavior of a grounded semi-infinite free space, and thus, a scalar potential will behave as $\left(1 / \rho^{2}\right)$ for high values of $\rho$. On the other hand, $T_{2}$ is always the dominant term in the near field, as already pointed out in [2], and becomes in a lossless case a pure real quantity. It is also interesting to note that the continuous-spectrum field $T_{1}+T_{2}$ behaves as $(1 / \rho)$ for small observation distances, and then evolves to the asymptotic $\left(1 / \rho^{2}\right)$ behavior for large distances. This should be expected, since close to the source the quasistatic behavior, which is $(1 / \rho)$ for the potentials, must be dominant.

Finally, the sum referred as $T_{3}$ corresponds to the set of surface waves generated by the multilayered medium. Their propagation constants are directly given by the values of the poles in the spectral plane, and they can interfere giving rise to sharp oscillations in the value of the Green's functions, as a function of the distance. It is currently said that surface waves are the dominant contribution in the far field region, but this is only true for strictly lossless structures. A non zero loss tangent will push the poles away from the real axis, and will introduce a small exponentially decreasing behavior in the Hankel functions appearing in $T_{3}$. As a result, the surface wave behavior $(1 / \sqrt{\rho})$ dominates only until a given distance. Then the terms devoid of exponential attenuation takes over, and the overall Green's function decreases as $\left(1 / \rho^{2}\right)$.

All the phenomena discussed above are clearly seen in Fig. 4(a) and (b), where the modulus of a typical scalar potential multilayered Green's function is depicted together with the partial contributions $T_{1}+T_{2}$ and $T_{3}$, as a function of the radial distance, for both the lossless and the lossy cases, respectively. In the lossless case the global behavior of the Green's function for $\left(k_{0} \rho>10\right)$ is $(1 / \sqrt{\rho})$ because the surface waves $\left(T_{3}\right)$ are dominant. For the lossy case, however, the surface waves are dominant only until a given distance, and then the sum $T_{1}+T_{2}$ takes over resulting in a global behavior of $\left(1 / \rho^{2}\right)$.

It is also interesting to see the behavior of the partial contributions of the surface wave poles excited in these structures. At the frequency of analysis there are three surface waves excited, and Fig. 5(a) presents the contribution of each one to the total potential. It can be seen that in all cases the behavior is of $(1 / \sqrt{\rho})$ type, and the interferences between them give rise to the sharp peaks

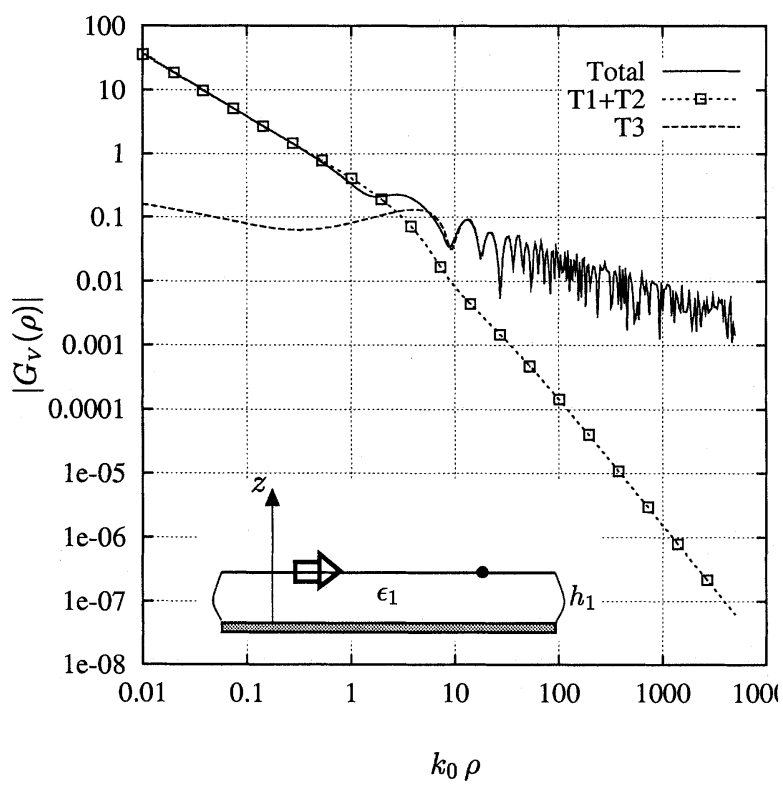

(a)

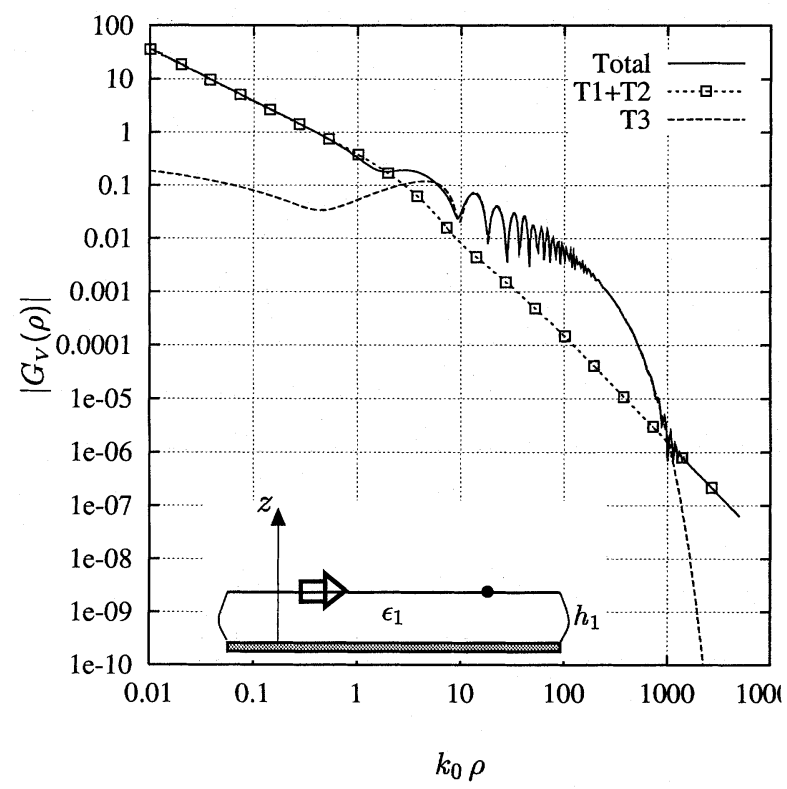

(b)

Fig. 4. Example of an electric scalar potential obtained for a lossless and a lossy structure using the new approach. In the lossy case the behavior for very large distances is $\left(1 / \rho^{2}\right) . \epsilon_{r_{1}}=4.4, h_{1}=10.0 \mathrm{~mm}$, frequency $10 \mathrm{GHz}$. (a) Lossless case: $\tan \delta_{1}=0.00$. (b) Lossy case: $\tan \delta_{1}=0.02$.

an oscillations in the response shown in Fig. 4(a). Furthermore, Fig. 5(b) presents the same surface wave contributions for the lossy substrate. It can be seen that for source-observer distances greater than $\left(k_{0} \rho=100\right)$, the negative exponential dominates the behavior of the surface wave terms, so that they exhibit a fast decaying behavior. This effect results in the overall $\left(1 / \rho^{2}\right)$ dependence of the potential due to the $T_{1}+T_{2}$ term, which takes over for very large source-observer distances.

There are special cases, however, where the continuous-spectrum field $T_{1}+T_{2}$ does not decay as $\left(1 / \rho^{2}\right)$ in the far field. This can occur when there is a surface wave pole very close to the branch point $k_{0}$. For the structure shown in Fig. 4(a) this 


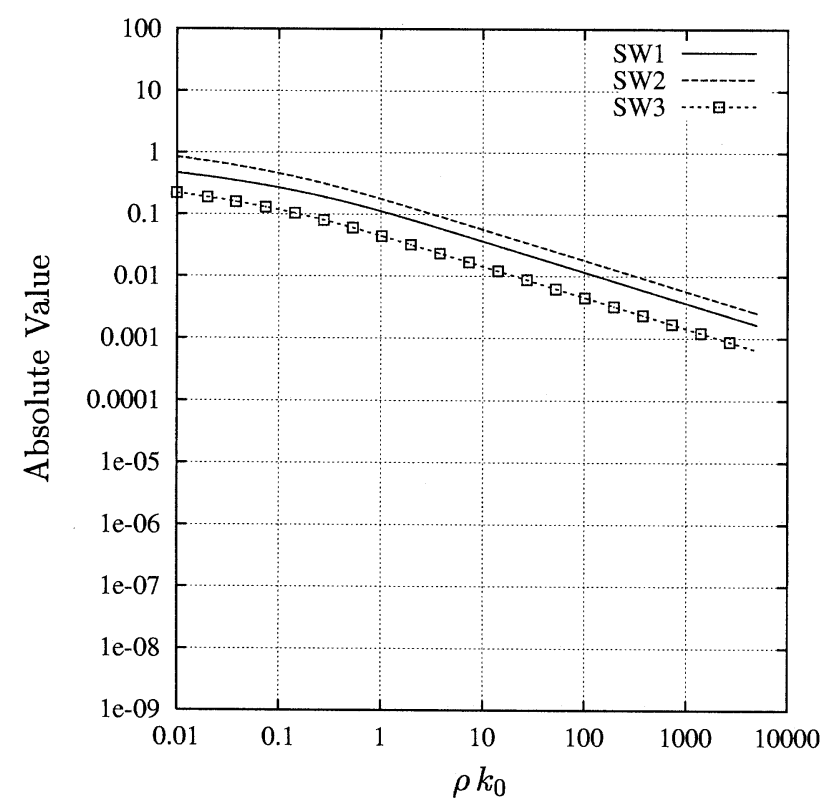

(a)

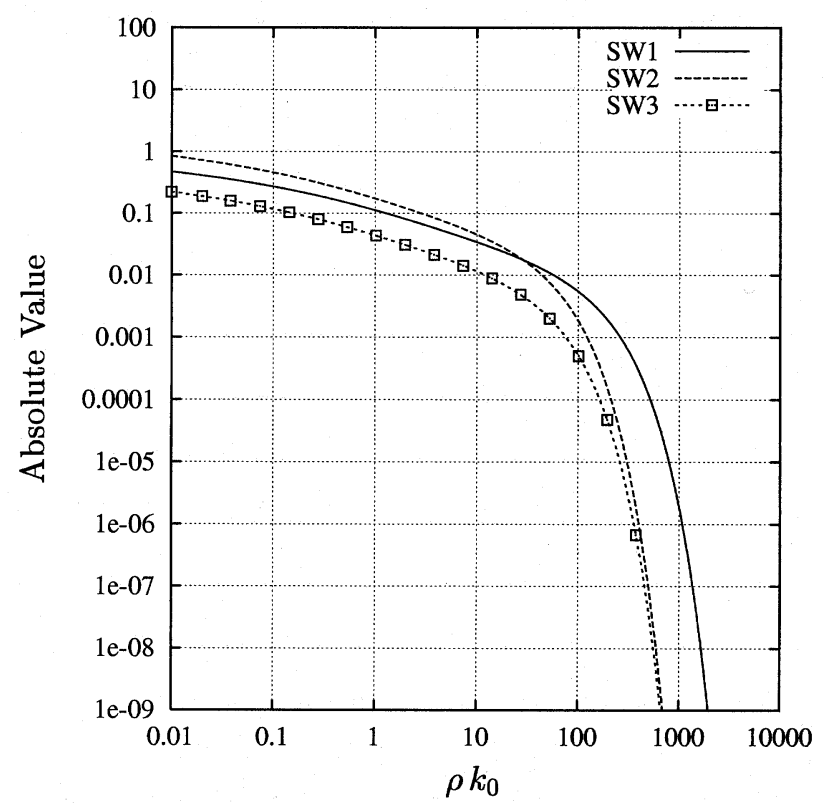

(b)

Fig. 5. Contribution of each surface wave pole to the final $T_{3}$ term, for the structure shown in Fig. 4. The lossy case clearly shows the strong attenuation effect on the surface waves for distances greater than $\left(k_{0} \rho=100\right)$. (a) Lossless case and (b) lossy case.

happens at a frequency $f=4.075 \mathrm{GHz}$ where a pole lies at $k_{\rho} / k_{0}=1.000027$. Fig. $6($ a) presents the different contributions of the imaginary axis formulation for this particular situation. It can be seen that the quasistatic term $T_{2}$ starts decreasing as $(1 / \rho)$ for small source-observer distances, and then it shifts to a $\left(1 / \rho^{2}\right)$ dependence in the far field region. This transition from a $(1 / \rho)$ dependence to a $\left(1 / \rho^{2}\right)$ dependence occurs around the distance $\left(\rho=1 / k_{0}\right)$. On its side, the term $T_{1}$ also experiences a transition, but this time from a $(1 / \sqrt{\rho})$ dependence to a $(1 / \rho)$ dependence. The transition occurs, as for the $T_{2}$ term, around the distance $\left(\rho=1 / k_{0}\right)$. It can also be observed that the whole

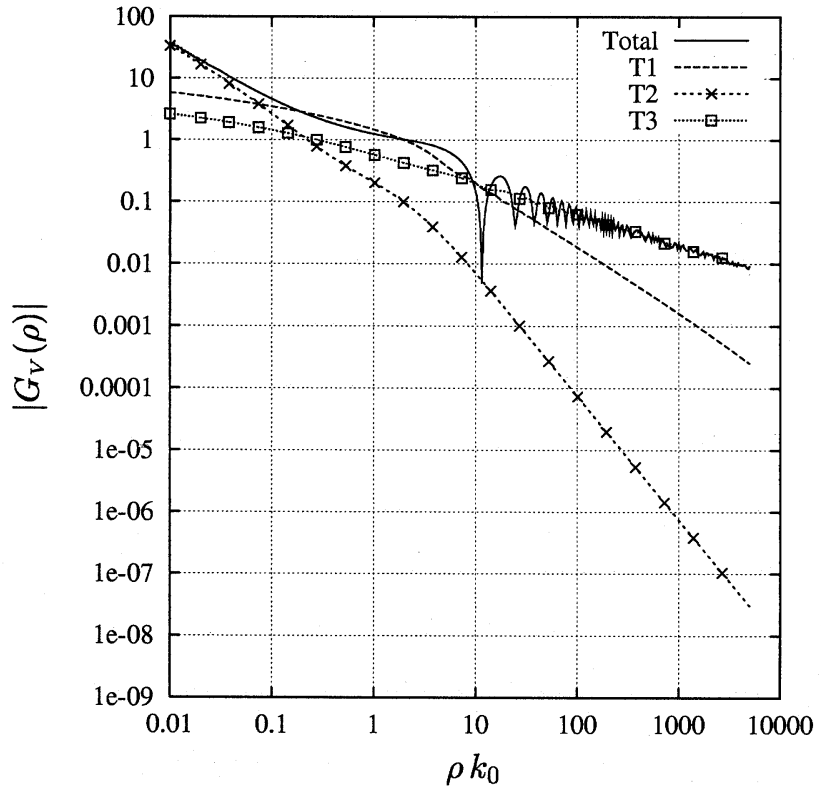

(a)

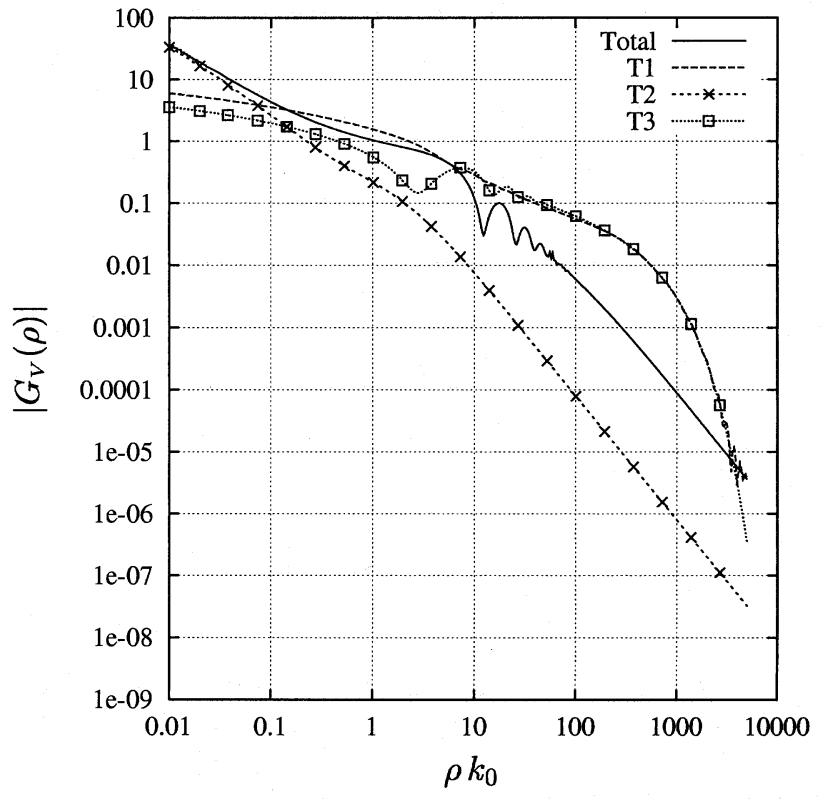

(b)

Fig. 6. Imaginary axis decomposition applied to the structure in Fig. 4 when there is a surface wave pole very close to the branch point. (a) Lossless case and (b) lossy case.

continuous-spectrum field varies as $(1 / \rho)$ for very large sourceobserver distances, and it is essentially due to the term $T_{1}$ (space wave term), since the term $T_{2}$ (quasistatic term) is negligible in comparison to the former.

An even more interesting situation can be observed for the lossy substrate, and when a surface wave pole is closed to the branch point. The imaginary axis decomposition for this case is shown in Fig. 6(b). It can be seen that now the space wave $T_{1}$ integral behaves in the far-field region exactly like the surface waves term $\left(T_{3}\right)$, including the exponential decaying behavior due to the losses in the substrate. In fact, due to the proximity of a surface wave pole to the branch point, the space wave term 


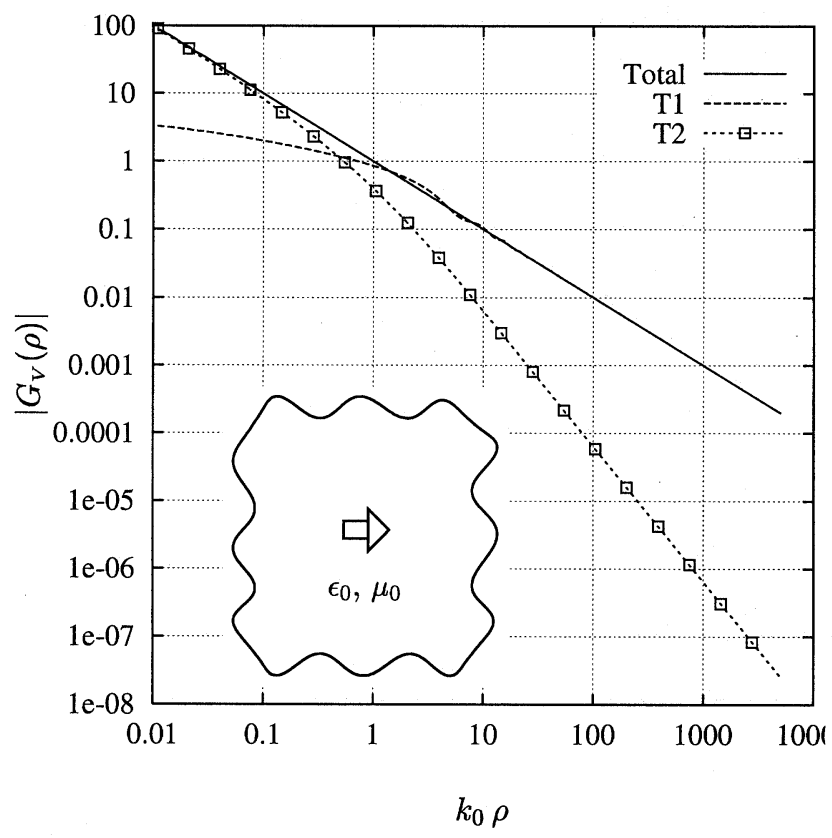

Fig. 7. Imaginary axis decomposition applied to an elementary dipole radiating in an unbounded free space medium.

associated to this branch point is now controlled by the surface wave pole. Interestingly, the contributions of both $T_{1}$ and $T_{3}$ terms combine together to give a total potential with $\left(1 / \rho^{2}\right)$ variation for large source-observer distances as shown in Fig. 6(b). It is also important to notice that the magnitude of both $T_{1}$ and $T_{3}$ terms are greater than the magnitude of the overall final potential. When they combine, their respective phases produce the required cancellation, therefore giving rise to a final potential with lower magnitude as clearly indicated in Fig. 6(b).

The behavior of the continuous-spectrum field in the lossless case can be consider of free-space type since a similar situation occurs for an elementary dipole radiating inside an unbounded free space medium (no ground plane). In this case, since there are no surface waves, the whole scalar potential behaves as $(1 / \rho)$. If the imaginary axis decomposition is used two different waves appear, namely the contribution of the space wave term $T_{1}$ behaving as $(1 / \rho)$, and the contribution of the $T_{2}$ integral which behaves in the far field region as $\left(1 / \rho^{2}\right)$. This fact is shown in Fig. 7 where we present the total scalar potential Green's function together with the partial contributions $T_{1}$ and $T_{2}$. Also, in Fig. 7 we can observe that the contribution due to the term $T_{2}$ is only dominant in the static near field region $(\rho \rightarrow 0)$. As the source-observer distance increases, the contribution $T_{2}$ is soon overcome by the space wave term $T_{1}$, thus resulting in the well known global behavior of $(1 / \rho)$. If we compare the $T_{1}$ and $T_{2}$ contributions obtained in the free-space configuration and in the inhomogeneous case [Fig. 6(a)], we can observe that they are very similar, and in particular the dependence with the radial distance is in both cases of the same type. For the inhomogeneous case, however, the global behavior of the potential is of $(1 / \sqrt{\rho})$ type due to the presence of two additional surface waves which are not present in the unbounded free space structure.

All above properties of the continuous spectrum field can be considered as the normal behavior for a source radiating in the

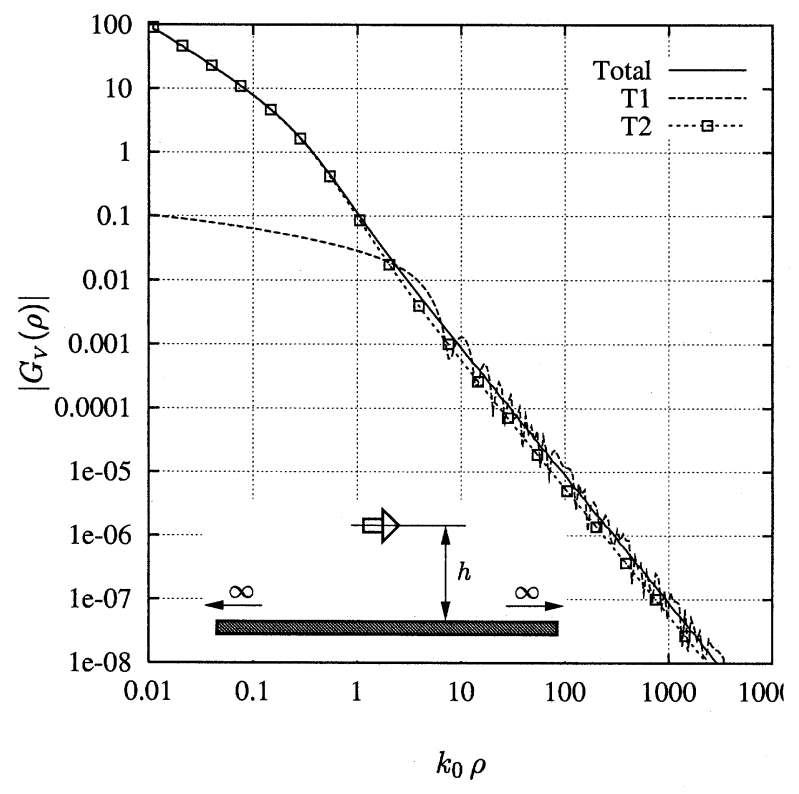

(a)

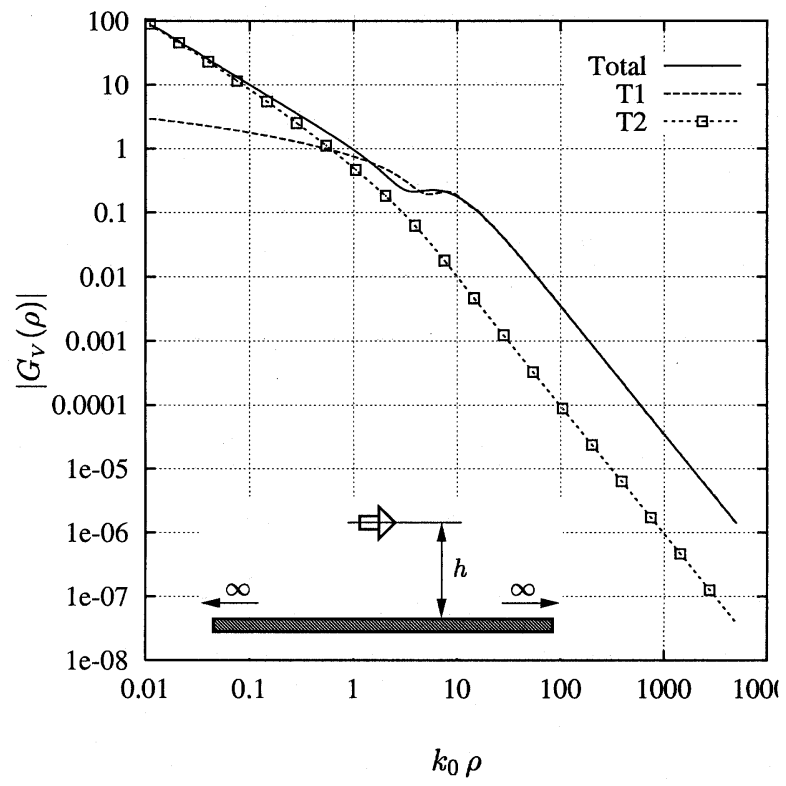

(b)

Fig. 8. Imaginary axis decomposition applied to an elementary dipole radiating in a grounded semi-infinite free space, for two different heights of the dipole with respect the ground plane. (a) $h=1 \mathrm{~mm}\left(\lambda_{0} / 30\right)$. (b) $h=$ $20 \mathrm{~mm}\left(2 \lambda_{0} / 3\right)$.

presence of an infinite ground plane. To show this we can take an elementary dipole inside a grounded semi-infinite free space environment. We know that in this case the total scalar potential behaves as $\left(1 / \rho^{2}\right)$ due to the interaction of the dipole and its image (and due to the absence of surface wave terms). If the new decomposition is used, the contributions associated to the terms $T_{1}$ and $T_{2}$ behave in the far field region as $\left(A / \rho^{2}\right)$ and $\left(B / \rho^{2}\right)$, respectively, where $A$ and $B$ are factors dependent on the electrical height of the dipole with respect the ground plane. The behavior in this case is clearly indicated in Fig. 8, where we show the total scalar potential together with the partial contributions $T_{1}$ and $T_{2}$ for a dipole at 1 and $20 \mathrm{~mm}$ distance from the ground plane. 
TABLE I

Asymptotic SPATIAL BEHAVIOR OF THE DIFFERENT COMPONENT OF GREEN's FUNCTIONS

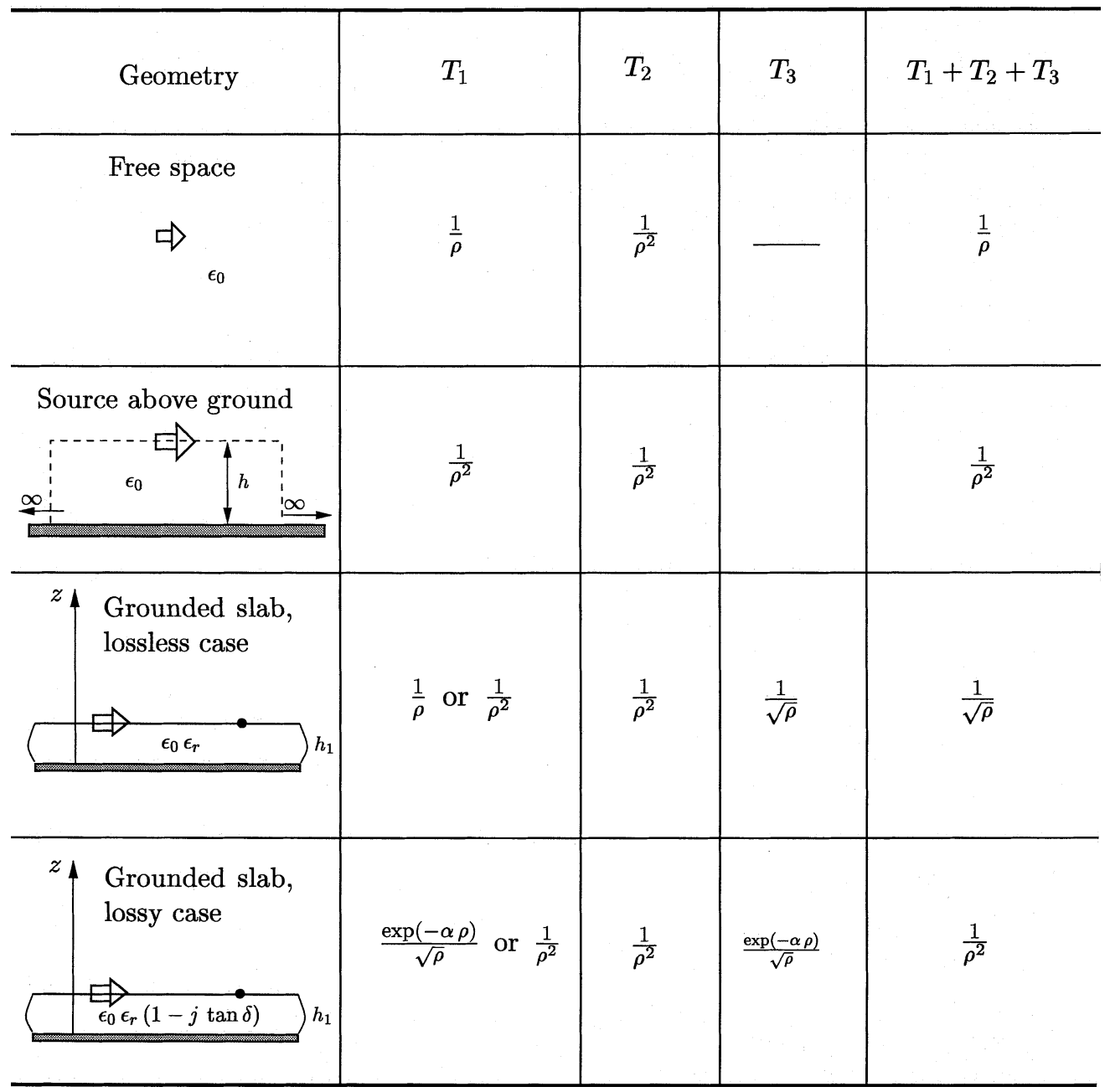

In the first case the contribution due to the term $T_{2}$ is closed to the space wave term, and thus, produces sharp oscillations in $T_{1}$. The sum of the two, however, gives in the far field region a smooth behavior with the well known $\left(1 / \rho^{2}\right)$ dependence. As regards the second case, the term $T_{2}$ is only dominant in the static near field region. As the source-observer distance increases, the term $T_{1}$ takes over and becomes the essential contribution to the total Green's function. As a summary, all these interesting spatial domain Green's functions asymptotic behaviors are collected in Table I, together with the partial contributions $T_{1}, T_{2}$, and $T_{3}$ introduced by the imaginary axis Sommerfeld integral decomposition.

\section{CONCLUSION}

In this paper an efficient method has been developed for the evaluation of Sommerfeld integrals. The method is based on choosing appropriate integration path contours which are closed through the imaginary axis of the complex plane. Following the proposed approach, the time consuming improper integrals involving highly oscillating Bessel functions along the real axis are transformed into improper integrals involving the fast decaying modified Bessel function $K_{n}$ along the imaginary axis. Due to this fast decaying behavior, the resulting integrals con- verge very fast, and can, therefore, be computed with less numerical effort.

The usefulness of the technique developed as a practical numerical tool resides on the ability of accurately locate the poles of the involved spectral Green's functions. For this purpose a general pole search algorithm has also been developed, and its general strategy briefly described.

With the developed approach, the special decomposition of the whole Green's functions in three partial terms has allowed to numerically study the asymptotic behavior of these Green's functions for large source-observer distances. Interesting new phenomena have been observed, specially in the lossy case. The proposed approach is expected to be very valuable in integral equation based CAD packages for the analysis and design of microstrip antennas and circuits.

\section{REFERENCES}

[1] K. A. Michalski and J. R. Mosig, "Multilayered media Green's functions in integral equations formulations," IEEE Trans Antennas Propagat., vol. 45, pp. 508-519, Mar. 1997.

[2] J. R. Mosig and F. E. Gardiol, "Analtical and numerical techniques in the Green's functions treatment of microstrip antennas and scatterers," Proc. Inst. Elect. Eng. Microwave Antennas and Propagation, vol. 130, pp. 175-182, Mar. 1983. 
[3] J. R. Mosig, Integral Equation Techniques, T. Itoh, Ed. New York: Wiley, 1989, Numerical techniques for microwave and millimeter-wave passive structures, ch. 3, pp. 133-213.

[4] R. C. Hall and J. R. Mosig, "The analysis of aperture coupled patch antennas via a mixed potential integral equation," IEEE Trans. Antennas Propagat., vol. 44, pp. 608-614, May 1996.

[5] N. G. Alexopoulos and D. R. Jackson, "Fundamental superstrate (cover) effects on printed circuit antennas," IEEE Trans. Antennas Propagat., vol. 32, pp. 807-815, Aug. 1984.

[6] J. M. Grimm and D. P. Nyquist, "Spectral analysis considerations relevant to radiation and leaky modes of open-boundary microstrip transmission line," IEEE Trans. Microwave Theory Tech., vol. 41, pp. 150-153, Jan. 1993.

[7] R. Shavit, "Dielectric cover effect on rectangular microstrip antenna array," IEEE Trans. Antennas Propagat., vol. 42, pp. 1180-1184, Aug. 1994.

[8] A. Hoorfar, J. X. Zheng, and D. C. Chang, "Numerical modeling of crossover and other junction discontinuities in two-layer microstrip circuits," Int. J. Microw. Millim. Wave Computer Aided Eng., vol. 2, no. 4, pp. 261-272, 1992

[9] P. Gay-Balmaz and J. R. Mosig, "3-d planar radiating structures in stratified media," Int. J. Microw. Millim. Wave Computer Aided Eng., vol. 7, pp. 330-343, 1997.

[10] M. Omid, M. Chiba, and M. Hayakawa, "Evaluation of microstrip Green function," Electron. Lett., vol. 33, pp. 434-435, Mar. 1997.

[11] Y. Brand, "Antennes Imprimées SSFIP: de l'élément Isolé au Réseau Planaire," Phd, Swiss Federal Institute of Technology, Laboratoire d'Electromagnetisme et d'Acoustique, Lausanne, Switzerland, 1996.

[12] P. R. Haddad and D. M. Pozar, "Anomalous mutual coupling between microstrip antennas," IEEE Trans. Antennas Propagat., vol. 42, pp. 1545-1549, Nov. 1994.

[13] A. A. Melcon and J. R. Mosig, "A novel spatial image technique for the analysis of shielded circuits and antennas," in Proc. 8th Biennial Conf. Electromagnetic Field Computation, IEEE CEFC'98, Tucson, AZ, June $1-3,1998$, p. 11

[14] M. A. Marin and P. H. Pathak, "An asymptotic closed-form representation for the grounded double-layer surface Green's function," IEEE Trans. Antennas Propagat., vol. 40, pp. 1357-1366, Nov. 1992.

[15] M. A. Marin, S. Barkeshli, and P. H. Pathak, "Efficient analysis of planar microstrip geometries using and closed-form asymptotic representation of the grounded dielectric slab Green's function," IEEE Trans. Microwave Theory Tech., vol. 37, pp. 669-679, Apr. 1989.

[16] Y. L. Chow, J. J. Yang, D. G. Fang, and G. E. Howard, "A closed form spatial Green's function for the thick microstrip substrate," IEEE Trans. Microwave Theory Tech., vol. 39, pp. 588-592, Mar. 1991.

[17] K. A. Michalski and J. A. Mosig, "Discrete complex image mpie analysis of a coax-fed coupled vertical monopoles in grounded dielectric substrate: Two formulations," in Proc. IEEE Microwave Antennas and Propagation, vol. 142, June 1995, pp. 269-274.

[18] R. Kipp, C. H. Chan, A. T. Yang, and J. T. Yao, "Simulation of high-frequency integrated circuits incorporating full-wave analysis of microstrip discontinuities," IEEE Trans. Microwave Theory Tech., vol. 51, pp. 848-854, May 1993.

[19] J. R. Mosig and T. K. Sarkar, "Comparison of quasistatic and exact electromagnetic fields from a horizontal electric dipole above a lossy dielectric backed by an imperfect ground plane," IEEE Trans. Microwave Theory Tech., vol. 34, pp. 379-387, Apr. 1986.

[20] L. B. Felsen and N. Marcuvitz, Radiation and Scattering of Waves. Englewood Cliffs, NJ: Prentice-Hall, 1973.

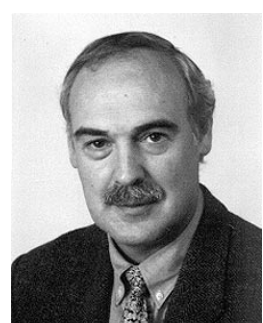

Juan R. Mosig (S'76-M'87-SM'94-F'99) was born in Cadiz, Spain. He received the electrical engineer degree from the Universidad Politecnica de Madrid, Madrid, Spain and the Ph.D. degree from Ecole Polytechnique Fédérale de Lausanne (EPFL), Switzerland, in 1973 and 1983, respectively.

In 1976, he joined the Laboratory of Electromagnetics and Acoustics, EPFL, Switzerland. Since 1991, he has been a professor at EPFL and since 2000, the Head of the EPFL Laboratory of Electromagnetics and Acoustics (LEMA). In 1984, he was a Visiting Research Associate at Rochester Institute of Technology, Rochester, NY. He has also held scientific appointments at universities of Rennes (France), Nice (France), the Technical University of Denmark and the University of Colorado at Boulder. He is the co-organizer and lecturer of yearly short intensive courses in Numerical Electromagnetics (Europe and USA). He is the author of four chapters in books on microstrip antennas and circuits and more than 100 reviewed papers. His research interests include electromagnetic theory, numerical methods and planar antennas.

Dr. Mosig is a Member of the Swiss Federal Commission for Space Applications, Chairman of a European COST project on antennas, and is responsible for several research projects for the European Space Agency.

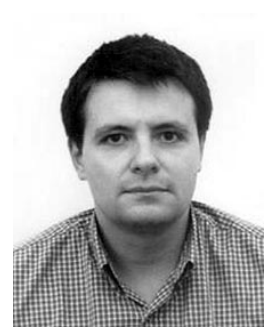

Alejandro Alvarez Melcón (M'98) born in Madrid, Spain, in 1965. He received the Ingeniero Superior de Telecomunicaciones degree from the Polytechnic University of Madrid (UPM), Madrid, Spain, in 1991 and the Ph.D. degree in electrical engineering from the Swiss Federal Institute of Technology, Lausanne, Switzerland, in 1998.

In 1988 he joined the Signal, Systems and Radiocommunications Department, UPM, as a Research Student, where he was involved in the design, testing, and measurement of broad-band spiral antennas for electromagnetic measurements support (EMS) equipment. From 1991 to 1993, he was with the Radio Frequency Systems Division, European Space Agency (ESA/ESTEC), Noordwijk, The Netherlands, where he was involved in the development of analytical and numerical tools for the study of waveguide discontinuities, planar transmission lines, and microwave filters. From 1993 to 1995, he was with the Space Division, Industry Alcatel Espacio, Madrid, Spain, and he worked at the ESA, where he collaborated in several ESA/ESTEC contracts. From 1995 to 1999, he was with the Swiss Federal Institute of Technology, École Polytechnique Fédérale de Lausanne, Lausanne, Switzerland, where he worked in the field of microstrip antennas and printed circuits for space applications. In 2000, he joined the Technical University of Carthagena, Cartagena, Spain, where he is currently developing his teaching and research activities.

Dr. Melcón received the JINA Best Paper Award for the best contribution to the JINA'98 International Symposium on Antennas and the COIT/AEIT Award to the best Ph.D. thesis in basic information and communication technologies. 